\title{
VARIATIONS GÉNÉTIQUES DU FONCTIONNEMENT HYPOPHYSAIRE DE TROIS SOUCHES DE RATTES IMMATURES. RELATIONS AVEC LA FERTILITÉ
}

\author{
P. MAULÉON et J. PELLETIER \\ avec la collaboration technique de Marie-Madeleine de Reviers, \\ Christine VIÉ et Françoise SIONNEAC \\ Laboratoire de Physiologie de la Reproduction, \\ Centre national de Recherches zootechniques, Jouy-en-Josas (Seine-et-Oise)
}

SOMMAIRE

L'étude du contenu hypophysaire en gonadotropine chez trois souches de Rattes Wistar âgées de 23 jours a été effectuée. Les déterminations des teneurs en FSH, par la technique de StEELMaN et PoHley, et de LH par celle de Parlow ont conduit aux résultats suivants :

- La teneur en FSH est moins élevée chez la souche $0_{3}(284,2$ équiv. $\mu \mathrm{g}$ FSH-NIHSi/mg de poudre antéhypophysaire sèche) que chez les souches o4 et I 2 (respectivement 555,9 et 596,7 équiv. $\mu \mathrm{g}$ FSH-NIHSI, $\mathrm{P}<0,0 \mathrm{I})$;

- La teneur en LH est la plus faible $(P<$ o,or $)$ chez la souche o4 (I 7,6 équiv. $\mu \mathrm{g}$ LH-NIHSI $/ \mathrm{mg}$ de poudre antéhypophysaire sèche contre $27, \mathbf{1}$ et 38,9 équiv. $\mu \mathrm{g}$ LH-NIHSI pour les souches 03 et 12 ).

Ces résultats sont discutés par rapport aux données de fertilité des trois séries de femelles reproductrices. En particulier la quantité de FSI déchargée dans le cas de la souche 12 et la faible sécrétion de LH dans celui de la souche $0_{4}$ semblent être les origines différentes de la faible fertilité de ces populations. Il est postulé en conséquence qu'il existe des variations génétiques de sécrétions et de décharges des gonadotropines hypophysaires chez les rattes de ces trois souches.

En outre les très fortes concentrations observées en FSII posent le problème de la spécificité des hormones gonadotropes.

\section{INTRODUCTION}

De grandes différences de fertilité ont été mises en évidence chez diverses souches de Rats. Elles peuvent provenir soit des possibilités de réponse de l'effecteur, soit de niveaux d'activités endocrines inégaux.

Des variations génétiques de sensibilité à l'action hormonale sont connues : pour la glande mammaire à l'hormone somatotrope (NANDI, I96I), la muqueuse 
vaginale aux oestrogènes (GARDNER et ARGyris, I957 ; TrEnTin, I950), le testicule à l'hormone stimulant l'interstitiel (LOSTROH, I962). Ces variations de sensibilité de l'effecteur sont dues dans certains cas à des variations de sa structure; ainsi celles de la sensibilité ovarienne à 1'hormone folliculo-stimulante sont liées à des différences de la population folliculaire (MAULÉon et RAO, I963).

En revanche, il a été rarement montré qu'une sécrétion hormonale puisse dépendre de facteurs génétiques : on sait par exemple qu'un fonctionnement thyroïdien très différent entre souris de diverses souches consanguines et hybrides a été relevé (AmIN et al., I957). Mais EDwards (I962) a montré que la réponse primaire à la sélection sur la taille de souris avait porté sur la taille de l'hypophyse plutôt que sur sa concentration en gonadotropines.

Les souches de rattes que nous avons étudiées au point de vue fertilité, sensibilité et structure ovariennes constituaient un matériel intéressant pour reprendre une telle étude.

\section{MATÉRIEL, ET TECHNIQUES}

Les animaux testés sont des rattes de race Wistar appartenant à trois souches développées au C. N. R. Z. désignées par les numéros 03 , 04 et I 2. Elles proviennent de trois couples appartenant au départ à trois souches consanguines. A partir de chacun de ces couples a été développée une colonie fermée dans laquelle un taux de consanguinité élevé a été maintenu.

L'abattage est effectué à 23 jours et les hypophyses sont aussitôt prélevées et plongées dans l'acétone pure redistillée. FSH et LH sont dosées sur un ensemble de 50 hypophyses pour chaque souche, chaque dosage étant répété deux fois.

\section{a) DOSAGE DE L'HORMONE FOLLICULO-STIMULANTE (FSH)}

Le dosage est effectué selon la technique de Steelman et Pohley (1953) sur des rattes Wistar 03 âgées de 23 jours. La poudre hypophysaire acétonique est administrée par voie sous-cutanée dans du sérum physiologique, selon trois doses, à raison de 6 rattes par dose en même temps que 20 III au total de HCG par ratte. Deux d'entre elles sont comparées au standard (FSH-NIHSI) injecté lui-même à deux niveaux. L'analyse statistique est celle décrite par EMMens (I948) et BLISS (I952); les résultats sont exprimés en équivalent- $\mu \mathrm{g}$ du standard FSH-NIHSr parmg de poudre acétonique hypophysaire sèche.

\section{b) Dosage de L'HoRmone Luténisante (L,H)}

La technique de Parlow (1958) modifiée par l'un d'entre nous (Pelletier, I963) a été appliquée à des rattes Holtzman (élevage Duterme) pour le dosage de LH. Les poudres hypophysaires des trois souches sont extraites pendant 4 heures par le sérum physiologique, avec agitation magnétique. Une seule extraction étant apparue suffisante, deux doses de surnageant sont administrées par voie intraveineuse à des lots de 6 à 8 rattes parallèlement à deux doses de LH-NIHSr qui servent de référence. L’acide ascorbique ovarien est estimé par la méthode de Mindlin et ButLer (I937). Les résultats sont exprimés en équivalent- $\mu$ g LH-NIHS I par mg de poudre sèche hypophysaire par comparaison des pentes de droites de régression de la poudre hypophysaire et du standard utilisé. Ces comparaisons sont effectuées après correction due au poids ovarien par analyse de covariance.

\section{RÉSULTATS}

La teneur en FSH par milligramme de poudre hypophysaire sèche est significativement plus faible dans les hypophyses des rattes des souches 03 et celle en $\mathrm{LH}$ est significativement plus faible pour les animaux des souches 04 comparées à celles des hypophyses des deux autres souches (tabl. I et 2 , fig. I et 2). 
TABLEAU I

Concentration en $L H$ des hypophyses de trois souches de rattes Wistar.

\begin{tabular}{|c|c|c|c|c|c|}
\hline $\begin{array}{l}\text { Souche } \\
\text { et Standard }\end{array}$ & $\begin{array}{c}\text { Nombre } \\
\text { d'animaux }\end{array}$ & $\begin{array}{l}\text { Dose } \\
(\mu \mathrm{g})\end{array}$ & $\begin{array}{c}\text { Ac. ascorbique } \\
\text { ovarien } \pm S \mathrm{~m} \\
\quad(\mu \mathrm{g})\end{array}$ & $\begin{array}{c}\text { Équiv. LH-NIH/mg } \\
\text { de poudre } \\
\text { hypophysaire } \\
(\mu g)\end{array}$ & $\begin{array}{l}\text { Intervalle } \\
\text { de confiance } \\
\vdots \quad(95 \%)\end{array}$ \\
\hline 03 & $\begin{array}{l}13 \\
13\end{array}$ & $\begin{array}{l}6,25 \\
25\end{array}$ & $\begin{aligned} 108,5 & \pm 2,94 \\
87,9 & \pm 1,86\end{aligned}$ & 27,1 & $\underbrace{18,4-39,8}$ \\
\hline 04 & $\begin{array}{l}13 \\
13\end{array}$ & $\begin{array}{r}25 \\
100\end{array}$ & $\begin{array}{l}93,3 \pm 2,21 \\
72,7 \pm 2,31\end{array}$ & $17,6^{* *}$ & $11,6-27,1$ \\
\hline 12 & $\begin{array}{l}13 \\
13\end{array}$ & $\begin{array}{l}6,25 \\
25\end{array}$ & $\begin{array}{r}102,2 \pm 3,75 \\
83,7 \pm 3,41\end{array}$ & 38,9 & $25,9-58,3$ \\
\hline LH-NIH & $\begin{array}{l}13 \\
13 \\
13\end{array}$ & $\begin{array}{l}0,2 \\
0,8 \\
3,2\end{array}$ & $\begin{array}{r}106,6 \pm 4,37 \\
84,7 \pm 3,92 \\
63,0 \pm 3,07\end{array}$ & & \\
\hline
\end{tabular}

** Teneur hautement significativement différente de celle de la souche 12.

$\lambda=0,305$.

\begin{tabular}{|c|c|c|c|c|c|}
\hline \multicolumn{6}{|c|}{ Concentration en FSH des hypophyses de trais souches de rattes Wistar } \\
\hline $\begin{array}{l}\text { Souche } \\
\text { et Standard }\end{array}$ & $\begin{array}{l}\text { Nombre } \\
\text { d'animaux }\end{array}$ & $\begin{array}{r}\text { Dose } \\
(\mu g)\end{array}$ & $\begin{array}{c}\text { Poids des deux } \\
\text { ovaires } \frac{1}{ \pm} \mathrm{Sm} \\
(\mathrm{mg})\end{array}$ & $\begin{array}{c}\text { Équiv. FSH-NIH/mg } \\
\text { de poudre } \\
\text { hypophysaire } \\
(\mu g)\end{array}$ & $\begin{array}{c}\text { Intervalle } \\
\text { de confiance } \\
(95 \%)\end{array}$ \\
\hline 03 & $\begin{array}{ll}6 \\
6 . & -.6\end{array}$ & $\begin{array}{l}300 \\
600\end{array}$ & $\begin{array}{r}67,2 \pm 6,73 \\
102,5 \pm 10,35\end{array}$ & $281,2 * *$ & $189,6-126,0$ \\
\hline $0_{t}^{\prime}$ & $\begin{array}{l}6 \\
6\end{array}$ & $\begin{array}{l}150 \\
300\end{array}$ & $\begin{array}{r}62,1 \pm 3,14 \\
105,1 \pm 8,11\end{array}$ & 555,9 & $482,0-656,0$ \\
\hline 12 & $\begin{array}{l}6 \\
6\end{array}$ & $\begin{array}{l}150 \\
300\end{array}$ & $\begin{array}{r}57,5 \pm 4,48 \\
114,5 \pm 10,17\end{array}$ & 596,7 & $446,6-633,2$ \\
\hline FSH-NIH & $\begin{array}{l}6 \\
6\end{array}$ & $\begin{array}{l}100 \\
200\end{array}$ & $\begin{array}{r}73,5 \pm 6,27 \\
114,2 \pm 10,53\end{array}$ & & \\
\hline
\end{tabular}

Teneur hautement' significativement différente de celle des souches 0 t et 12 . Index de précision $\lambda \rightleftharpoons 0,132$. 


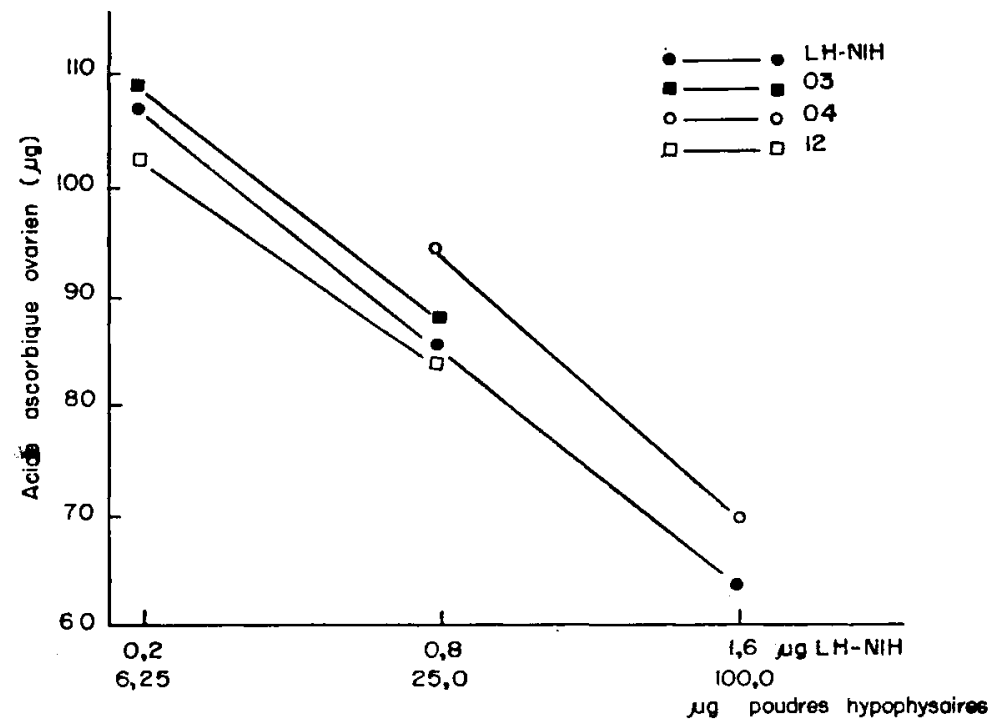

FIG. 1. - Diminution de l'acide ascorbique ovarien a l'injection dhe standard LH-NIHS1 et des poudres hypophysaires des souches 03,04 et $\mathrm{I}_{2}$

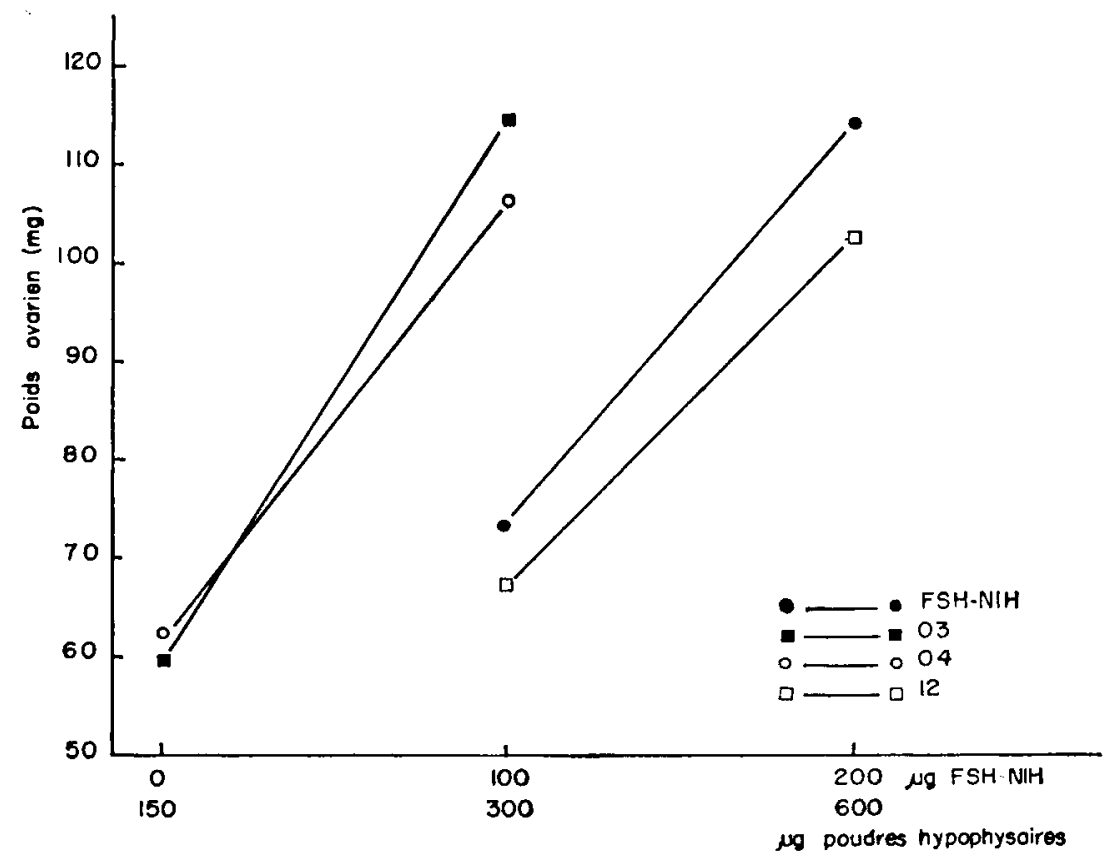

FIG. 2. - Croissance du poids ovarien a l'administration du standard FSH-NIHS1 et des poudres hypophysaires des souches 03,04 et $\mathrm{I}_{2}$

Le poids sec moyen de l'hypophyse des rattes de la souche $\mathrm{r} 2$ étant de $0,5 \mathrm{r} \mathrm{mg}$ et celui des rattes des souches 04 et 03 de $0,48 \mathrm{mg}$, les fortes concentrations enFSH et LH des hypophyses de la souche 12 correspondent bien à une teneur totale plus élevée en hormones gonadotropes. 
En revanche, on peut dire que les animaux de la souche 04 ne disposent que de $50 \mathrm{p}$. Ioo de la potentialité hypophysaire en $\mathrm{L} H$ des rattes des deux autres souches et ceux de la souche 03 que de 50 p. Ioo de la potentialité en FSH.

\section{DISCUSSION}

\section{I. - RELATIONS ENTRE LES TENEURS RELATIVES EN FSH ET LH} DES HYPOPHYSES DES RATTES DES TROIS SOUCHES ETT LEUR FERTILITÉ

La fertilité des animaux des trois colonies développées à partir des couples 03, 04 et $\mathbf{I} 2$ se situe à des niveaux différents pour la taille de la portée, le pourcentage de femelles stériles, le pourcentage d'accouplements fertiles et surtout pour le nombre de jeunes rats nés par femelle fertile, pendant une période identique. La souche la plus fertile est incontestablement la souche 03 ; les causes de faible fécondité des souches 04 et 12 ne semblant pas être identiques (tabl. 3).

\section{TABLEAU 3}

Fertilité des trois souches de rattes 03 , 04, I 2

(Les résultats de ce tableau ont été tirés des documents d'élevage de la raterie du C. N. R. Z. pour I961-62 et I962-63; effectif de 250 femelles en reproduction pour la souche 03 et de 70 pour les souches $0_{4}$ et 12 ).

\begin{tabular}{|c|c|c|c|c|c|c|c|c|}
\hline \multirow{3}{*}{ Souche } & \multirow{3}{*}{$\begin{array}{l}\text { Poids moyen } \\
\text { au sevrage } \\
\pm \mathrm{Sm}(\mathrm{g})(\mathrm{l})\end{array}$} & \multirow{3}{*}{$\begin{array}{c}\text { Taille } \\
\text { de la portée } \\
\pm \text { Sm (2) }\end{array}$} & \multicolumn{3}{|c|}{$\begin{array}{c}\% \text { de femelles }\left({ }^{3}\right) \\
\text { stériles/femelles accouplées }\end{array}$} & \multirow{3}{*}{$\begin{array}{l}\text { Nombre } \\
\text { d'accou- } \\
\text { plements } \\
\text { pour } \\
\text { 1re saillie } \\
\text { fertile (4) }\end{array}$} & \multirow{2}{*}{\multicolumn{2}{|c|}{$\begin{array}{l}\begin{array}{l}\text { Nombre total } \\
\text { de petits nés } \\
\text { par femelle }\end{array} \\
\text { Durée } \\
\text { d'utilisation de }\end{array}$}} \\
\hline & & & \multirow{2}{*}{$\begin{array}{c}\text { au } 1^{\text {er }} \text { cycle } \\
\text { de repro- } \\
\text { duction }\end{array}$} & \multirow{2}{*}{$2^{e} \begin{array}{l}\text { cucle } \\
\text { cycle }\end{array}$} & \multirow{2}{*}{$\begin{array}{c}\text { au } \\
3^{\mathrm{e}} \text { cycle }\end{array}$} & & & \\
\hline & & & & & & & $200-250 \mathrm{j}$ & $250-300 \mathrm{j}$ \\
\hline $0_{4}^{\prime}$ & $45,0 \pm 0,56$ & $6,47 \pm 0,15$ & $x^{\prime} \mathbf{t}, 1$ & 32,8 & 44,2 & $2,91 \pm 0,56$ & 12,67 & 12,00 \\
\hline 12 & $38,7 \pm 0,53$ & $8,88 \pm 0,1_{1}^{\prime}$ & 32,6 & 20,7 & 28,8 & $3,45 \pm 0,52$ & $23,4^{\prime}$ & 25,69 \\
\hline 03 & $47,4 \pm 0,43$ & $9,65=0,07$ & 25,1 & 15,1 & 13,2 & $3,01 \pm 0,95$ & 29,08 & 36,10 \\
\hline
\end{tabular}

(1) Sur cent jeunes rattes pour chaque souche.

(2) Sur les trois premières portées pour chaque femelle fertile.

$\left({ }^{3}\right)$ Les femelles sont considérées comme stériles si non gestantes après plus de 8 accouplements.

(4) Seulement sur femelles fertiles.

a) La souche I2 est de fertilité moyenne pour trois raisons

- Le pourcentage d'animaux stériles est de l'ordre de $30 \mathrm{p}$. Ioo et surtout se maintient à ce niveau au cours de chaque cycle de reproduction ;

- la taille des portées est significativement plus faible que celle de la souche o3; 
- la production des femelles n'est pas accrue par une durée d'utilisation supérieure à 225 jours.

Le vieillissement génital rapide de cette souche peut être dû à un épuisement de son stock de follicules primordiaux que nous savons faible à 23 jours, 1'ovaire de ces rattes immatures possédant alors une faible population de follicules en croissance et de follicules à antrum (Mauléon et HaNumantha RaO, I963).

Il est paradoxal qu'un si petit nombre de follicules existe dans l'ovaire à cet âge alors que la teneur hypophysaire en FSH et LH est élevée et que la croissance de ces follicules dépend des gonadotropines hypophysaires comme le prouve l'évolution quantitative de cette population folliculaire après hypophysectomie à cet âge (Benoit et Mauléon, I964).

Deux hypothèses peuvent être avancées : ou bien, il y a absence de décharge en FSH chez les animaux de cette souche, ou bien sécrétion et décharge sont très élevées ce qui entraîne, entre la naissance et 23 jours, un épuisement rapide de la réserve folliculaire. Un fait d'observation est en faveur de cette deuxième hypothèse : le taux d'atrésie des follicules en croissance et des follicules à antrum est de 43 p. roo dans les ovaires de rattes de 23 jours de cette souche alors qu'il n'est que de 20 p. Ioo dans ceux des rattes de la souche 04 .

\section{b) La faible fertilité de la souche 04 est caractérisée}

- par un pourcentage d'animaux stériles de plus de 40 p. Ioo au cours de tous les cycles de reproduction;

- par une faible taille des portées;

- par un nombre total de portées très faible au cours de la vie sexuelle.

La petite taille et la rareté des portées de cette souche peuvent être la conséquence soit d'un faible taux d'ovulation, soit de difficultés d'implantation des oufs ou d'une mortalité embryonnaire très forte.

L'ovaire de ces rattes possède une population folliculaire bien développée en rapport avec une sécrétion hypophysaire en FSH " normale " mais la teneur en LH des hypophyses de ces rattes à 23 jours est relativement faible.

Il est logique d'associer ce dernier fait aux causes possibles de faible fertilité. En effet, si cette teneur en LH est basse cela peut résulter soit d'une décharge précoce, soit d'une faible sécrétion qui se retrouvera chez les animaux adultes ovulant mal et lutéinisant mal les follicules éclatés. Des observations préliminaires sur la teneur en progestérone des corps jaunes des animaux de cette souche sont en faveur de cette hypothèse.

\section{c) La souche 03 est fertile et le demeure} pendant une période supérieure à 275 jours

Le développement normal d'une population folliculaire ovarienne de taille moyenne et une teneur élevée en $\mathrm{LH}$ des hypophyses de rattes de 23 jours cadrent avec cette fertilité durable. Une faible potentialité hypophysaire en FSH à cet âge s'explique mal, à moins qu'elle ne soit la conséquence d'une décharge précoce importante de cette hormone. Il est indispensable qu'une étude cinétique de l'évolution en fonction de l'âge de ces hormones soit faite pour que les explications des relations fertilités et sécrétions endocrines soient plus faciles et qu'une telle hypothèse reçoive des arguments en sa faveur. 
Néanmoins il nous semble qu'il existe des variations génétiques de sécrétions et de décharges en gonadotropines hypophysaires chez les rattes de ces trois souches.

FowLER et EDWARDS ( 1960 ) pensaient à de telles variations à la suite de comparaisons du taux d'ovulations induites par diverses doses de PMSG avec celui observé après un accouplement normal chez les souris des souches $\mathrm{N}$ et $\mathrm{C}$ sélectionnées en lignées d'après leur taille. Si EDwards (I962) n'a pu le démontrer par dosage des gonadotropines sur l'utérus de souris immature, il est possible que la technique utilisée soit en cause.

\section{II. - VAleurs ABSOLUES DES CONCENTRATIONS EN FSH ET LH} DES HYPOPHYSES DE RATTES IMMATURES ET SPÉCIFICITÉ DES HORMONES

Les concentrations en $\mathrm{L}_{4} \mathrm{H}$, comprises entre I $_{5}$ et $40 \mu \mathrm{g}$ équiv. $\mathrm{L}_{4} \mathrm{H}-\mathrm{NIHSI}$, se situent dans le même ordre de grandeur que celles trouvées par SchwARTz et BARTOSIK (I g62) et par Gorski et BarRaClough ( I962).

Les concentrations en FSH, entre 280 et 596 équiv. $\mu$ g FSH-NIHSi, apparaissent particulièrement fortes. Il ne peut s'agir d'une erreur d'estimation puisque chez la Ratte mature, PARLow (I964) trouve une teneur équivalente (I60 $\mu \mathrm{g}$ FSH-NIHSI).

Bien que la richesse de 1'hypophyse du Rat en FSH ait été montrée bien supérieure à celle d'autres espèces telles que le Porc, le Mouton ou le Cheval (PAyNe et al., I959), il est difficile de concevoir que l'hormone folliculo-stimulante représente la moitié du poids sec de l'hypophyse. Nous pensons donc que la teneur hypophysaire déduite d'une comparaison entre un standard d'origine ovine et une hormone, provenant d'une même espèce que l'effecteur ovarien est surestimée. Un tel fait pose donc le problème de la spécificité des hormones gonadotropes.

De même il n'est pas exclu qu'une souche présente une plus grande sensibilité à sa propre hormone, et que l'utilisation de la souche 03 pour les estimations comparatives des teneurs hypophysaires en FSH des trois souches constitue une erreur de méthodologie. Nous étudierons l'influence de ce facteur dans un prochain travail.

$$
\text { Reçu pour publication en mars } 1964 .
$$

\section{SUMMARY}

GENETIC VARIATION IN HYPOPHYSEAI, FUNCTION IN THREE STRAINS OF IMMATURE RATS. RELATION TO FERTILITY

Gonadotropin content of the hypophysis of 3 strains of female Wistar rats 23 days old was studied. Estimation of follicle stimulating hormone (FSH) by the method of Steelman and Pohley, and of luteinising hormone (LH) by the method of Parlow, gave the following results :

The content of FSH was significantly less $(\mathrm{P}<0.01)$ in strain 03 ( 284.2 equiv. $\mu g$ FSH-NIHS $1 / \mathrm{mg}$ dried prehypophysis) than in strains $0_{4}$ and $\mathrm{I} 2$ ( 555.9 and 596.7 equiv. $\mu$ g FHS-NIHSr, respectively).

The content of LH was least $(\mathrm{P}<0,0 \mathrm{r})$ in strain 04 ( 7.6 equiv. $\mu g$ LH-NIHSr/mg dried pre. hypophysis compared with 27.1 and 38.9 equiv. $\mu$ g LH-NIHSi for strains 03 and 12).

The results are discussed in relation to data on fertility of 3 series of breeding females. In particular the output of FSH by strain I 2 and the small secretion of IH in strain 04 seem to be the separate reasons for the poor fertility of those populations. It is postulated, consequently, that these strains of rats differ genetically in the secretion and output of hypophyseal gonadotropins.

Also, the high concentrations of FSH raise the problem of the specificity of gonadotropic hormones. 


\section{REMERCIMENTS}

Nous remercions le service "Endocrinology study section at the National Institutes of Health " qui nous a aimablement fourni les hormones hautement purifiées FSH-NIH-SI et LH-NIH-SI.

\section{RÉFÉRENCES BIBLIOGRAPHIQUES}

Amin A., Cirai C. K. et Reineke E. P., 1957. Differences in thyroid activity of several strains of mice and $\mathrm{F}_{1}$ hybrids. Amer. J. Physiol, 191, 34-36.

Benolt MI., MaulÉon P., 1964. (Communication personnelle).

Busss C. I., 1952. The statistics of bioassay with special reference to the vitamins. Acad. Press. New York.

EDWARDS R. G., 1962. The size and endocrine activity in mice selected for large and small body size. Genet. Res. Canb., 3, 428-443.

Emmens C. W., I948. Principles of biological assay. Chapman and Hall. London.

Fowler R. E., EDwards R. G., I g6o. The fertility of mice selected for large and small body size. Genet. Res. Camb., 1, 397-407.

Gardner W. U., ARgYris B. F., I957. Differences in vaginal sensitivity of mice of in-bred strains; localization in the vagina. Endocrinology, 60, 532-546.

Gorski R. A., Barraclougi C. A., i962. Adeno-hypophyseal LH content in normal androgen-sterilized and progesterone primed sterile female rats. Acta Endocr., 30, I3-2 I.

LostroH A. J., 1962. Effect of testosterone and growth hormone on nucleic acid and protein in the sex accessory glands of Long-F,vans and Sprague-Dawley rats. Endocrinology, 70, 747-749.

Mauléon P., Hanumantha Rao K., 1963. Variations génétiques des populations folliculaires dans les ovaires de rattes impubères. Ann. Biol. anim. Bioch. Biophys., 3, 2I-3I.

Mindin R. L., Butler A. M., 1937. The determination of ascorbic acid in plasma. J. Biol. Chem., $122,673-686$.

NANDI S., I96r. Differential responsiveness of $A$ and $C_{3} H$ mouse mammary tissues to somatotrophin containing hormone combinaison. Proc. Soc. Exp. Biol. Med., 108, 1-3.

Parlow A. F., 1958. A rapid bioassay for $\mathrm{LH}$ and factors stimulating $\mathrm{LH}$ secretion. Feder. Proc., 17, 402.

PARLOW A. F., 1964. Importance of differential quantitative bioassays for pituitary gonadotrophins in the rat. Endocrinology, 74, 1 38-14I.

Payne R. W., Runser R. H., Hagans J. A., Morrison R. D. Assay of folliculo-stimulating hormone in the hypophysectomized cestrogen treated immature female rat. Endocrinology, 65, 389-394.

Pelletier J., 1963. Étude critique du dosage de ICSH par la méthode de l'acide ascorbique ovarien. Ann. Biol. anim. Bioch. Biophys., 3, 307-323.

Schwartz N. B., Bartosik D., I962. Changes on pituitary LH content during the rat estrous cycle. Endocrinology, 71, 756-762.

Steelman S. L., Pohley F. M., 1953. Assay of the follicle stimulating hormone based on the augmentation with human chorionic gonadotrophin. Endocrinology, 53, 604-6I6.

TRENTIN J. J., I950. Vacrinal sensitivity to ostrogen as related to mammary tumor incidence in the mice. Cancer Res., 10, $580-583$. 